\title{
Propositions pour la mise en place d'un enseignement interprofessionnel entre étudiants sages-femmes et internes de médecine générale à l'Université de Lyon
}

\author{
Proposals for the implementation of an interprofessional training course for \\ midwifery and general practitioner students at Lyon university
}

\author{
Charline BOISSY ${ }^{1}$, Marion LAMORT-BOUCHÉ ${ }^{1,2}$, Yves ZERBIB ${ }^{1,2}$, Elisabeth SIMON $^{3}$ \\ et Marie FLORI ${ }^{1}$ \\ 1 Collège Universitaire de Médecine Générale, Université Claude Bernard Lyon 1, Lyon, France \\ 2 EA 4148 Sciences et Société - Historicité, Éducation, Pratiques (S2HEP), Université Claude Bernard Lyon 1, Lyon, France \\ 3 Faculté de médecine et de maïeutique Charles Merieux, Université Claude Bernard Lyon 1, Lyon, France
}

\author{
Mots-clés \\ Interne de médecine \\ générale ; \\ étudiant en \\ maïeutique ; \\ enseignement ; \\ coopération inter- \\ professionnelle ; \\ compétences
}

Résumé - Contexte : La loi HPST (2009) introduit le suivi gynécologique de prévention et la prescription de contraception en dehors de la grossesse dans les compétences des sagesfemmes. Médecins généralistes et sages-femmes ont des compétences communes décrites pour chaque profession dans un référentiel métier et compétences. Il convient de connaître et d'articuler ces compétences dès la formation initiale pour exercer ensuite en coopération. But : Recueillir les propositions des enseignants et enseignés de maïeutique et de médecine générale de l'université de Lyon à propos d'un futur enseignement commun entre étudiants des deux filières. Méthodes : Une étude qualitative exploratrice dans le champ de la planification pédagogique a été conduite à l'aide de groupes de discussion focalisée et d'entretiens individuels semi-structurés. Les participants étaient respectivement des enseignants et des enseignés des deux filières de l'université. Les thèmes explorés étaient les étudiants concernés, le moment pour le réaliser, le contenu, la forme pédagogique, l'encadrement, la fréquence et l'objectif attendu. Résultats : Cet enseignement interprofessionnel se baserait sur des cas cliniques inspirés de familles de situations cliniques issus des référentiels métiers et compétences. Il concernerait les étudiants en fin de cursus. Le travail s'effectuerait en petits groupes encadrés par un binôme médecin généraliste et sage-femme, formés à la pédagogie et ayant une activité clinique. L'enseignement comprendrait : la présentation des deux professions, une analyse de pratiques à partir de cas cliniques rapportés et une synthèse. L'intérêt serait de mieux connaître le champ de compétences des deux professions. Conclusion : Cette étude définit les caractéristiques d'un enseignement interprofessionnel de maïeutique et médecine générale, qui puisse répondre aux attentes de chacune des filières qui se connaissent peu. 


$\begin{array}{ll}\begin{array}{l}\text { Keywords } \\ \text { General practice }\end{array} & \text { Abstract - Background: Gynecological preventive monitoring and the prescription of } \\ \text { residency; midwife } & \text { contraceptives are part of midwives' areas of expertise since it was adopted in the so-called } \\ \text { student; teaching; } & \text { HPST act in France. General practitioners and midwives have common skills with respect to } \\ \text { inter-professional } & \text { the monitoring of women's health. These skills are outlined in the occupational and skill refe- } \\ \text { collaboration; } & \text { rence guidelines. One needs to know and organize these skills in order to work collaborati- } \\ \text { competencies } & \text { vely. Goal: The aim is to collect propositions from teachers and students in view of setting up } \\ \text { a common teaching program for midwives and interns in General Practice (GP) in Lyon. } \\ \text { Methods: We conducted an exploratory qualitative study in pedagogical planning through } \\ \text { focus groups and interviews. Participants were selected among teachers and students from } \\ \text { both streams in the UCBL1 university. Explored topics addressed questions such students } \\ \text { involved (the right time to offer the program, previous internships), the content, the educatio- } \\ \text { nal format, the supervision, the frequency and the expected objectives for the program. } \\ \text { Results: This interprofessional program would be based on clinical cases that draw on gene- } \\ \text { ral clinical situations stemming from occupational and skill reference guidelines. It would } \\ \text { involve students who are at the end of their studies. Applicants would work in small groups } \\ \text { supervised by a GP and a midwife who both have a background in pedagogy and are involved } \\ \text { in clinical activities. The program would include a presentation of both professions, an analy- } \\ \text { sis of the practices based on clinical cases and a synthesis. The ultimate goal would be provi- } \\ \text { ding broader and deeper knowledge of the areas of expertise of each profession. Conclusion: } \\ \text { This study outlines the main features of an interprofessional teaching program for GPs and } \\ \text { midwives that would meet the expectations of two professions that do not know each other } \\ \text { very well. }\end{array}$

\section{Introduction}

Adoptée le 21 juillet 2009, la loi portant réforme de l'hôpital et relative aux patients, à la santé et au territoire (loi dite HPST) a fait évoluer le champ de compétence des sages-femmes en France. Le code de santé publique permet à cette profession médicale de prendre en charge le suivi gynécologique et la prévention de la femme en bonne santé pendant et en dehors de la grossesse, y compris en matière de vaccination et de contraception (articles L4151-1 ; L4151-2 et L4151-4). Ainsi les sages-femmes, en plus du suivi de la femme enceinte, peuvent prescrire et réaliser les vaccins, mettre en œuvre toutes les contraceptions (hormonale, par dispositif souscutané ou intra-utérin) et assurer le suivi de dépistage en réalisant des frottis cervico-utérin pour toutes les femmes en bonne santé tout au long de leur vie y compris en dehors de la grossesse. Ces missions rejoignent celles du médecin généraliste (article L4130-1 du code de santé publique) : le médecin généraliste peut lui aussi réaliser les frottis cervico-utérins de dépistage, prescrire et mettre en œuvre toutes les contraceptions, suivre le statut vaccinal de ses patientes et également assurer le suivi de la grossesse jusqu'au sixième mois.

En 2010 la mission "Evaluation des compétences professionnelles des métiers de la santé »a publié dans un même ouvrage le référentiel métier et compétences des médecins généralistes et celui des sages femmes ${ }^{[1]}$. Ces référentiels ont pour objectif de clarifier les contenus de chaque métier. Ils confirment les compétences énoncées par le code de santé publique.

Ces compétences identiques, exercées par deux professions différentes, présentent un levier pour améliorer la santé de la femme en France en augmentant les portes d'entrées dans le système de santé. Pour cela, un travail interprofessionnel entre ces deux professions médicales peut permettre d'améliorer la prise en charges des femmes. Pour exercer ainsi, il convient à ces deux professions de connaître et d'articuler ces compétences communes pour travailler en synergie. 
Le Centre for the Advancement of Interprofessional Education (CAIPE) a défini en 2002 les formations interprofessionnelles comme des formations où « deux professions ou plus apprennent avec, de et sur l'autre, pour améliorer la collaboration et la qualité des soins ${ }^{[2]}$. Le Consortium pancanadien sur l'interprofessionnalisme en santé (CIHCPIS) adhère à cette définition et souligne qu'une formation interprofessionnelle efficace contribue à améliorer la qualité des soins et rehausse la pratique de chaque profession concernée $^{[3]}$. L'Organisation mondiale de la santé (OMS) rappelle en 2010 l'importance d'une formation interprofessionnelle pour assurer par la suite un exercice collaboratif. Elle émet un certain nombre de recommandations pour assurer la réussite de tels programmes avec notamment l'utilisation de méthodes d'apprentissage qui utilisent des expériences pratiques des étudiants et où l'interaction se produit entre les étudiants ${ }^{[4]}$.

La formation initiale est le premier temps où peuvent être abordés les champs de compétences identiques ou complémentaires des différents professionnels qui seront amenés à travailler ensemble ${ }^{[5]}$. Toutefois à l'issue de cette formation, les internes de médecine générale semblent être nombreux à ne pas connaître l'ensemble des compétences des autres professions et plus particulièrement les compétences communes. Cette connaissance semble pourtant nécessaire à une prise en charge efficiente des patientes. Les stages ambulatoires en soins primaires peuvent être l'occasion de rencontrer ces professionnels, mais à l'heure actuelle de façon peu formalisée. Fortes de cette analyse, de plus en plus de facultés de médecine en France ont développé des séminaires interprofessionnels permettant de développer cette réflexion. Le plus souvent ces séminaires concernent les professions de santé les plus connues des étudiants (pharmaciens, kinésithérapeutes...). Depuis trois ans, des communications dans les congrès du Collège national des généralistes enseignants mettent en avant ces séminaires. Mais la littérature ne rapporte pas d'expériences d'enseignement commun ayant pour point de départ explicite les similitudes ou les interfaces des référentiels métiers et compétences des sages-femmes et des médecins généralistes, à savoir la prise en charge de la femme en bonne santé, en situation de grossesse ou non, dans le champs de la prévention (dépistage, vaccination, contraception) ainsi que le suivi du début de grossesse.

L'objectif de ce travail est de rapporter la préparation d'un enseignement entre les étudiants sagefemme et les internes de médecine générale s'articulant autour des compétences identiques à ces deux professions. Pour que ces notions soient abordées sous une forme adaptée aux deux professions en formation, nous avons recueilli les propositions de la population concernée (les enseignants et les enseignés). La finalité était de mettre en place, lors de la formation initiale, un enseignement commun s'organisant autour de ces compétences partagées ou communes.

\section{Méthodes}

Une étude qualitative exploratoire dans le champ de la planification pédagogique à été réalisée.

La population cible est celle visée par l'enseignement à mettre en place : les étudiants sages-femmes, les internes de médecine générale et les enseignants de ces deux filières à l'Université Claude Bernard Lyon 1.

Des groupes de discussion focalisée (focus groups) d'internes de médecine générale (tableau I) et d'étudiants sages-femmes (tableau II) ont été réalisés de façon séparée. Cette séparation a été décidée dans le but de permettre une expression libre de chacun à propos de l'autre profession. Des étudiants sage-femme en dernière année (cinquième année) ont été interrogés. Ce moment du cursus correspond à l'année professionnalisante, au cours de laquelle ils peuvent réaliser un stage ambulatoire pour compléter leur formation selon les modalités antérieures à la réforme des études de maïeutique de 2010. Des internes de médecine générale ayant validé le stage de niveau 1 chez le médecin généraliste ou ayant réalisé plus de la moitié de ce stage ont été interrogés. A ce stade, ils ont dépassé le niveau novice des compétences du métier de médecin généraliste ${ }^{[6]}$. Le recrutement a été fait parmi les étudiants sages-femmes et les internes inscrits à l'université Claude Bernard 
Tableau I. Caractéristiques des étudiantes sages-femmes ayant participé au groupe de discussion focalisée.

\begin{tabular}{|l|c|c|c|c|c|c|}
\hline & Age & Projet professionnel & Sexe & Stage ambulatoire & $\begin{array}{c}\text { Structure } \\
\text { du stage }\end{array}$ & $\begin{array}{c}\text { Durée du } \\
\text { groupe de } \\
\text { discussion }\end{array}$ \\
\hline ESF1 & 23 & Autre $:$ ce qu'il y aura & F & Bientôt & Non applicable & Cabinet seul \\
\hline ESF2 & 23 & Hospitalier & F & Bientôt & Cabinet seul \\
\hline ESF4 & 22 & $\begin{array}{c}\text { Hospitalier puis } \\
\text { ambulatoire }\end{array}$ & F & Bientôt & Cabinet seul \\
\hline ESF5 & 25 & $\begin{array}{c}\text { Hospitalier puis } \\
\text { ambulatoire }\end{array}$ & F & Bientôt & Cabinet seul \\
\hline ESF6 & 23 & $\begin{array}{c}\text { Hospitalier puis } \\
\text { ambulatoire }\end{array}$ & F & Oui & Cabinet seul & \\
\hline
\end{tabular}

ESF : Etudiante sages-femmes

$\mathrm{F}:$ Féminin

Tableau II. Caractéristiques des internes de médecine générale ayant participé au groupe de discussion focalisée.

\begin{tabular}{|c|c|c|c|c|c|c|c|c|}
\hline & \multirow{2}{*}{ Age } & \multirow{2}{*}{ Sexe } & \multirow{2}{*}{$\begin{array}{c}\text { Projet } \\
\text { professionnel }\end{array}$} & \multirow{2}{*}{ Stage en cours } & \multicolumn{3}{|c|}{ Stages validés } & \multirow{2}{*}{$\begin{array}{l}\text { Durée du } \\
\text { groupe de } \\
\text { discussion }\end{array}$} \\
\hline & & & & & MG & GP & SASPAS & \\
\hline IMG1 & 27 & $\mathrm{~F}$ & Ambulatoire & Non concerné & $\mathrm{x}$ & & $\mathrm{x}$ & \multirow{5}{*}{$1 \mathrm{~h} 47 \mathrm{~min}$} \\
\hline IMG2 & 28 & $\mathrm{~F}$ & NSP & SASPAS & $\mathrm{X}$ & & & \\
\hline IMG3 & 27 & $\mathrm{~F}$ & Ambulatoire & GP & $\mathrm{x}$ & & & \\
\hline IMG4 & 27 & $\mathrm{M}$ & Ambulatoire & Hospitalier & $\mathrm{X}$ & & & \\
\hline IMG5 & 28 & $\mathrm{~F}$ & Ambulatoire & Non concerné & $\mathrm{X}$ & & & \\
\hline IMG6 & 29 & $\mathrm{~F}$ & Mixte & SASPAS & $\mathrm{X}$ & & & \multirow{5}{*}{$1 \mathrm{~h} 29 \mathrm{~min}$} \\
\hline IMG7 & 27 & $\mathrm{~F}$ & NSP & $\mathrm{MG}$ & & & & \\
\hline IMG8 & 28 & $\bar{F}$ & Ambulatoire & SASPAS & $\mathrm{X}$ & & & \\
\hline IMG9 & 28 & $\mathrm{~F}$ & NSP & $\mathrm{MG}$ & & & & \\
\hline IMG10 & 27 & $\mathrm{~F}$ & Ambulatoire & SASPAS & $\mathrm{X}$ & & & \\
\hline
\end{tabular}

F : Féminin

$\mathrm{M}:$ Masculin

GP : Gynécologie - pédiatrie

IMG : Interne de médecine générale

MG : Médecine générale

SASPAS : Stage ambulatoire de soins primaires en autonomie supervisée

Lyon 1. Il s'est effectué par l'envoi de courriels aux personnes répondant aux critères d'inclusion. Un échantillonnage de disponibilité et d'intérêt à été constitué à partir de l'entretien avec les répondants.

Des entretiens semi-structurés d'enseignants des deux filières (tableaux III et IV), considérés comme experts, ont été réalisés. Ces enseignants ont été recrutés parmi les enseignants sage-femme et de médecine générale de Lyon. Un échantillonnage raisonné des enseignants a été constitué, avec recherche de la variation maximale concernant leurs rôles et fonctions universitaires. Les enseignants ont été interrogés en 
Tableau III. Caractéristiques des enseignantes sages-femmes interrogées.

\begin{tabular}{|c|c|c|c|c|c|c|c|}
\hline & Sexe & $\begin{array}{c}\text { Ancienneté en } \\
\text { enseignement }\end{array}$ & $\begin{array}{c}\text { Activité de } \\
\text { Soins }\end{array}$ & $\begin{array}{c}\text { Type } \\
\text { d'exercice }\end{array}$ & $\begin{array}{c}\text { Structure } \\
\text { libérale }\end{array}$ & $\begin{array}{c}\text { Durée } \\
\text { d'exercice }\end{array}$ & $\begin{array}{c}\text { Durée des } \\
\text { entretiens }\end{array}$ \\
\hline SF1 & F & 7 ans & Actuelle & Libéral & seule & 23 ans & $40 \mathrm{~min}$ \\
\hline SF2 & F & 21 ans & Passée & Hospitalier & NC & 13 ans & 46 min \\
\hline SF3 & F & 22 ans & Passée & Hospitalier & NC & 09 ans & $52 \mathrm{~min}$ \\
\hline
\end{tabular}

SF : Sage-femme

$\mathrm{F}$ : féminin

$\mathrm{NC}$ : non concernée

Tableau IV. Caractéristiques des enseignants de médecine générale ayant participé aux entretiens semi-structurés.

\begin{tabular}{|c|c|c|c|c|c|}
\hline & Sexe & $\begin{array}{c}\text { Anciennenté en } \\
\text { enseignement }\end{array}$ & $\begin{array}{c}\text { Lieu } \\
\text { d'exercice }\end{array}$ & $\begin{array}{c}\text { Durée } \\
\text { d'exercice }\end{array}$ & $\begin{array}{c}\text { Durée } \\
\text { d'entretiens }\end{array}$ \\
\hline MG1 & F & $\mathbf{1 4}$ ans & Urbain sensible & $\mathbf{2 5}$ ans & $\mathbf{2 2 ~ m i n ~}$ \\
\hline MG2 & M & 28 ans & Semi-rural & 37 ans & 38 min \\
\hline MG3 & M & 30 ans & Urbain & 34 ans & 33 min \\
\hline MG4 & M & 21 ans & Urbain sensible & 25 ans & 23 min \\
\hline
\end{tabular}

F : Féminin

$\mathrm{M}:$ Masculin

MG : Médecin généraliste

entretiens individuels en raison de contraintes liées à leur manque de disponibilité et pour leur permettre une expression libérée des masques sociaux habituellement présents dans les échanges en groupe de statuts différents.

Un guide d'entretien a été rédigé en s'inspirant de la méthode «Qui ? Quoi ? Où ? Quand ?

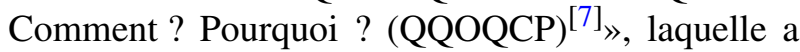
ensuite permis une catégorisation thématique lors de l'analyse des verbatims. Le même guide d'entretien a été utilisé pour les groupes de discussion focalisée et les entretiens semi-dirigés. Les entretiens ont débuté par le rappel aux enseignants et aux étudiants des grandes lignes de l'autre cursus (formation pratique et théorique). Puis les thèmes abordés ont été :

- les étudiants concernés ;

- les compétences identiques et les thèmes à aborder ;

- le lieu où réaliser l'enseignement ;

- le moment dans les deux cursus (médecine générale et sage-femme) où le réaliser ;

- la logistique à mettre en place ;

- l'intérêt d'un tel enseignement.
Les groupes de discussion focalisée et les entretiens ont été enregistrés avec l'accord des participants.

Après retranscription intégrale et anonymisation, les verbatims ont été analysés à l'aide du logiciel NVivo 10 en suivant les catégorisations thématiques définis par le guide d'entretien et également de façon inductive. Les données ont été analysées par trois chercheurs.

\section{Résultats}

Le recueil a eu lieu du 24 janvier au 5 septembre 2013. Nous avons réalisé un groupe de discussion focalisée d'étudiants sages-femmes, trois entretiens d'enseignants sages-femmes, deux groupes de discussion focalisée d'internes de médecine générale et quatre entretiens d'enseignants de médecine générale. Les groupes de discussion focalisée ont duré entre $1 \mathrm{~h} 04$ et $1 \mathrm{~h} 47$ et les entretiens entre 22 et 52 minutes. 
Les caractéristiques de la population, les codes correspondant dans les verbatims et les durées des entretiens et des groupes de discussion focalisée sont détaillées dans les tableaux I, II, III et IV.

Les enseignants de médecine générale interrogés exercent au sein d'un cabinet libéral de groupe. Les enseignants sages-femmes interrogés sont des femmes et ont toutes eu une activité urbaine hospitalière ou libérale avant d'enseigner. Une seule exerce encore une activité libérale alors que les deux autres sont exclusivement enseignantes.

\section{Méconnaissance de l'autre profession}

Les internes de médecine générale ont confirmé le postulat initial de leur méconnaissance du champ de compétences des sages-femmes : « je ne sais pas exactement ce qu'elles font » (IMG1).

Ce sentiment est partagé par certains de leurs enseignants : «Ce qui est flou pour moi c'est ... ce qu'est le métier de sage-femme » (MG2).

Cette mauvaise connaissance de leur métier est soulignée par les étudiants sage-femme : «Que ce soit les externes ou les internes en premier semestre déjà ils disent ... vous pouvez faire tout ça...» (ESF3).

Les étudiants en sage-femme pensent connaître le métier de médecin généraliste (MG) : « nous on se doute bien de ce dont ils sont capables mais eux ils se doutent moins de ce dont on est capable. » (ESF3).

\section{Attentes}

Les objectifs identifiés sont de «décloisonner pendant les études médicales c'est une urgence » (1A). Il s'agit aussi de pouvoir se connaître, « pouvoir revenir sur la notion de partage et d'échange pour voir ce qu'on a en commun et nos différences aussi » (EMG4) et ce afin de «Comprendre ce que fait l' autre effectivement » (2B). La finalité étant de « désigner des tâches communes (qui) vont déterminer des rôles et des compétences communes » (EMG4).

\section{Propositions vis à vis de l'enseignement}

Les participants ont d'abord exprimé l'envie d'aborder des savoirs ou des savoir-faire (pose de stérilet, allaitement, ...) avant de mettre l'accent sur les compétences et notamment celle de «Education en santé dépistage ; prévention individuelle et communautaire » et $«$ professionnalisme ${ }^{[8]}$.

L'analyse des verbatims a conduit à dégager des propositions vis à vis de l'enseignement à mettre en place. La saturation des données a été obtenue pour le contenu, le lieu, le moment pour les étudiants, l'encadrement et l'organisation pédagogique. Dans un deuxième temps les tendances dégagées et les consensus nous ont permis de définir l'organisation de l'enseignement projeté : à savoir un enseignement interprofessionnel ayant pour contenu les compétences identiques aux professions de sages-femmes et de médecins généralistes. L'organisation retenue à l'issue de l'analyse des verbatims est présentée dans le tableau V.

Enfin l'activité professionnelle a été évoquée avec la volonté pour les étudiants sages-femmes et les internes de médecine générale de travailler dans des structures de groupe et le plus souvent pluri-professionnelles.

\section{Discussion}

Force et limites méthodologique du travail

\section{Concernant le recueil des données}

La méthode utilisée pour recueillir les données a permis à tous les étudiants sages-femmes et tous les internes de médecine générale qui ont répondu aux courriels de participer. Mais cette méthode n'a recueilli que les avis de ceux qui ont montré un intérêt pour le sujet.

Les critères d'inclusion définis par la méthode ont été respectés et seuls les étudiants et internes avancés dans le cursus professionnel ont été interrogés. Ayant dépassé le stade novice, ils ont une conscience plus marquée de l'importance d'une formation professionnalisante. Leurs propositions vont d'ailleurs dans le sens d'un enseignement professionnalisant tourné vers leur future pratique. Ce choix a exclu les étudiants en début de cursus, qui sont davantage naïfs 
Tableau V. Caractéristiques du projet d'enseignement commun.

\begin{tabular}{|c|c|c|}
\hline Qui & Obligatoire ou optionnel & Obligatoire \\
\hline Quoi & Le contenu & $\begin{array}{l}\text { Prise en charge conjointe dans le champ des compétences } \\
\text { communes }\end{array}$ \\
\hline Où & Le lieu & Lieu facultaire sage-femme ou médecine \\
\hline \multirow[t]{3}{*}{ Quand } & Moment pour les SF & Dernière année \\
\hline & Moment pour les IMG & Pendant le stage chez le médecin généraliste \\
\hline & Fréquence & 2 fois \\
\hline \multirow[t]{3}{*}{ Comment } & Encadrement & $\begin{array}{l}\text { Binôme d'enseignants mixte formé à la pédagogie et ayant une } \\
\text { activité clinique }\end{array}$ \\
\hline & pédagogique & $\begin{array}{l}\text { - pré requis fourni en amont } \\
\text { - cas clinique et analyse de pratique } \\
\text { - possible mise en situation }\end{array}$ \\
\hline & Organisation & $\begin{array}{l}1 \text { journée avec valorisation des moments de détente } \\
\text { petits groupes mixtes } \\
\text { En trois temps : } \\
\text { - présentation des deux professions } \\
\text { - cas cliniques et analyse de pratique } \\
\text { - mise en commun des idées fortes de chaque groupe }\end{array}$ \\
\hline Combien & L'effectif & Groupes mixtes de 10 à 20 personnes \\
\hline
\end{tabular}

SF : Sages-femmes

IMG : Internes de médecine générale

sur le contenu de leur cursus car ils ne l'ont pas encore expérimenté.

Un échantillonnage raisonné a été constitué au regard des différents statuts d'enseignants représentés à la faculté de médecine de Lyon et dans l'école de sages-femmes. Les enseignants de médecine générale conservent un exercice de soins, leur statut universitaire leur imposant de conserver cette activité. Parmi les enseignantes sages-femmes une seule conservait un exercice de soins en libéral : elle était maître de stage et accueillait des étudiants. Les autres enseignants de l'école de sages-femmes avaient un poste d'enseignement à plein temps.

Le type de recrutement n'a pas permis d'interroger d'hommes étudiants ou enseignants sagesfemmes qui sont très minoritaires. Seul un interne homme a participé.

L'usage de la méthode QQOQCP indexée par le CNRS a permis de répondre de manière systématique à l'ensemble des questions conduisant à la planification d'un enseignement.

\section{Concernant la méthode d'analyse}

Les verbatims des groupes de discussion focalisée et des entretiens individuels ont été analysés ensemble. L'analyse a été effectuée à l'aide du logiciel NVivo. L'usage d'un logiciel permet un partage du projet. Ce logiciel a été choisi car connu et maîtrisé par l'ensemble des chercheurs.

La saturation des données a été obtenue sur le contenu, l'encadrement, la population concernée, l'organisation, le lieu et le but de cet enseignement et a permis de conduire à une proposition d'enseignement. Nous n'avons pas obtenu la saturation complète des données sur les craintes. Cela est le reflet de la diversité des personnes interrogées. Pour atteindre la saturation dans ce domaine il aurait fallut pouvoir interroger davantage de personnes et notamment recruter des personnes non-intéressées par le sujet.

La méthode utilisée a permis une triangulation des participants (enseignants et enseignés) et des sources (médecins généralistes et sages-femmes) 
pour définir les grandes lignes d'un enseignement commun.

La triangulation des chercheurs a permis de modérer le biais d'interprétabilité.

\section{Crédibilité, fiabilité, transférabilité}

La saturation des données sur les thèmes traitants de la mise en place de l'enseignement confère un degré de crédibilité à ce travail. Cette crédibilité aurait été renforcée par l'obtention de la saturation à propos des craintes et des attentes. Les participants n'ont pas eu la possibilité de valider les retranscriptions des verbatims. Toutefois les conclusions du travail ont été envoyées à chaque participant et certains ont participé à l'enseignement qui a ensuite été mis en place. Ils ont pu exprimer leur adhésion aux résultats.

Les résultats ont conduit à planifier un enseignement qui reprend les modèles pédagogiques actuellement utilisés centrés sur l'aprenant ${ }^{[10]}$. Cette cohérence retrouvée est une force de ce travail.

Enfin les résultats sont facilement transférables dans d'autres facultés. Ce travail propose un modèle d'enseignement répondant à des attentes et pouvant facilement être mis en place dans d'autres régions.

\section{Discussion des résultats}

\section{L'enseignement}

L'enseignement interprofessionnel vise à se développer partout dans le monde et est de plus en plus décrit ${ }^{[9]}$. Les exemples d'enseignements communs décrits dans la littérature s'attachent à permettre aux participants de découvrir une autre profession et ses champs propres de compétences ${ }^{[10]}$. N'ayant pas trouvé dans la littérature de modèle d'enseignement se basant sur des compétences identiques entre deux professions, le modèle proposé ici ne peut pas être comparé.

Le projet final extrait de ce travail tient compte de l'évolution de la réflexion des participants et retient les propositions finales faites par les groupes et individus interrogés. Les participants ont d'abord envisagé un enseignement qui se superpose à celui qu'ils connaissent puis ils ont proposé quelque chose de nouveau. Cela est très marqué chez certains qui proposaient des cours magistraux avant d'imaginer un enseignement pratique où ils sont acteurs. C'est une illustration du changement pédagogique observé ces dernières décennies. Il consiste en un passage du paradigme d'enseignement (où l'enseignant transmet des savoirs) à un paradigme d'apprentissage (où l'apprenant est l'acteur principal $)^{[11]}$. Dans ce contexte, l'outil pédagogique plébiscité est la mise en situation par des situations cliniques authentiques proposées par les apprenants. Cette organisation rejoint les recommandations $l^{\prime} \mathrm{OMS}^{[4]}$ qui recommande l'utilisation de méthodes d'apprentissage qui utilisent des expériences pratiques des étudiants, provoquant l'interaction entre les étudiants. Les problématiques que les participants souhaitent aborder correspondent à la situation autour de la sexualité et de la génitalité décrite dans les grandes familles de situations cliniques ${ }^{[12]}$ (situations concernant la prise en charge et suivi de la contraception, de la grossesse, de l'interruption volontaire de grossesse et le dépistage des cancers).

Enfin la volonté exprimée par les étudiants sagesfemmes et les internes de travailler dans des structures de groupes, le plus souvent pluri-professionnelles, est à mettre en lien avec le développement des réseaux qui contribuent à développer et favoriser ce genre d'exercice. Pourtant les réseaux ne sont pas abordés dans la formation médicale initiale. L'enseignement proposé pourrait être l'occasion d'aborder ce modèle d'exercice.

\section{La méconnaissance de l'autre profession}

Tous les participants ont relevé la méconnaissance de l'autre profession. Pour les personnes interrogées le fait d'organiser un enseignement autour de ces compétences doit permettre aux participants de mieux se connaître sans pour autant que cet enseignement commun s'articule autour de cette méconnaissance. Cela concorde avec les retours à deux ans des étudiants ayant participé à un enseignement interprofessionnel à Rennes ${ }^{[10]}$.

À l'heure actuelle, beaucoup de médecins généralistes et de sages-femmes travaillent « à côté » mais 
souhaitent de plus en plus travailler « avec » l'autre profession. Cette constatation est en accord avec les travaux réalisés ces dernières années sur le souhait des internes ${ }^{[13]}$ qui plébiscitent le travail en groupe, mais aussi sur la réalité observée comme le confirment le Conseil national de l'Ordre des médecins ${ }^{[14]}$ et Levy ${ }^{[15]}:$ «Le regroupement, formel, des spécialistes en médecine générale avec d'autres professionnels exerçant dans les soins de premiers recours (...) connaît un regain d'intérêt ».

\section{Conclusion}

Ce travail préliminaire à la mise en place d'un enseignement commun a permis d'en dégager les grandes lignes mais aussi d'identifier une méconnaissance mutuelle des deux professions. Les professionnels reconnaissent avoir des difficultés car ils n'ont pas « du tout appris à collaborer». Les futurs professionnels quant à eux confirment cette envie de travailler « avec » l'autre.

Le développement de structures ambulatoires rend effectif ce souhait de travailler ensemble (cabinets de groupe, mono ou pluri-professionnel, maisons et pôles de santé, centres de santés, réseaux, ...). La formation initiale intègre d'ailleurs ce nouveau mode de travail avec la mise en place d'enseignements interprofessionnels et de stages dans les maisons de santé. Cet enseignement qui insiste de plus en plus sur le travail pluri-professionnel contribuera probablement à développer ce mode d'exercice dans le futur.

Forte de ces constats, il convient à la formation initiale de préparer les futurs médecins généralistes et sages-femmes à leur exercice futur. Un enseignement offrant à ces deux professions un moment d'apprentissage et de réflexion commun, ainsi qu'une meilleure connaissance de l'autre profession, pourrait être un apport supplémentaire permettant aux futurs professionnels de travailler plus facilement ensemble.

\section{Contributions}

Charline Boissy a réalisé ce travail de recherche dans le cadre de la thèse pour le diplôme d'état de docteur en médecine. Elle a participé à la conception du protocole de recherche, au recueil des données, à l'analyse des résultats et à la rédaction du manuscrit. Marion Lamort-Bouché et Marie Flori ont participé à la conception du protocole de recherche, au recueil des données et à l'analyse des résultats. Elisabeth Simon et Yves Zerbib ont participé aux relectures successives du manuscrit.

\section{Déclaration d'intérêts}

Aucun des auteurs ne déclare de conflit d'intérêt en lien avec le contenu de cet article

\section{Approbation éthique}

Non sollicitée

\section{Valorisation scientifique}

Ce travail a fait l'objet :

- d'une communication orale au $13^{\text {ème }}$ Congrès du Collège national des généralistes enseignants (CNGE) à Clermont-Ferrand en novembre, 28 et 29 novembre 2013 ;

- d'une communication orale au $14^{\text {ème }}$ Congrès du Collège national des généralistes enseignants (CNGE) à Lille, 26-28 novembre 2014 ;

- d'une communication orale au $5^{\text {ème }}$ Congrès de la Société internationale francophone d'éducation médicale (SIFEM) à Bruxelles, 8-10 avril 2015.

\section{Références}

1. Collectif : Mission nationale, Evaluation des compétences professionnelles des métiers de la santé ; Collège National des Généralistes Enseignants (CNGE) ; Conseil National de l'Ordre des SagesFemmes (CNOSF); Collectif des Associations et Syndicats de Sages-Femmes (CASSF) ; Collège National des Gynécologues et Obstétriciens Français (CNGOF). Référentiel métier et compétences. Médecins généralistes, sages-femmes et gynécologues obstétriciens. Paris : Berger-Levrault, 2010. 
2. Centre For The Advancement Of Interprofessional Education (CAIPE). Definition of Interprofessional education. 2002. [On-line] Disponible sur : http:// www.caipe.org.uk/about-us/defining-ipe/.

3. Consortium pancanadien sur l'inter professionnalisme en santé (CIHCPIS). Définition et principes de la formation interprofessionnelles : la perspective du CPIS. 2007 [On-line] Disponible sur :

http://tools.hhr-rhs.ca/

index.php?option=com_mtree\&task=att_download \&link_id=6652\&cf_id=69\&lang=fr

4. World Health Organization. Framework for Action on Interprofessional Education and Collaborative Practice, 2010 [On-line] Disponible sur : http:// apps.who.int/iris/bitstream/10665/70185/1/ WHO_HRH_HPN_10.3_eng.pdf

5. Association nationale des étudiants sages-femmes (ANESF) \& Intersyndicale nationale autonome représentative des internes de médecine générale (ISNAR-IMG). Organisation de la prise en charge de la santé de la femme. 2011 [On-line]. Disponible sur : http://anesf.com/wp/wp-content/uploads/

Sant\%C3\%A9-de-la-femme-Contribution-commune-Anesf-ISNAR-IMG.pdf

6. Compagnon L, Bail P, Huez J, Stalnikiewicz B, Ghasarossian C, Zerbib Y et al. Les niveaux de compétences. Exercer 2013;108:156-64.

7. Valeins H. Réseau Qualité en Recherche - QQOQCP: Quoi, Qui, Où, Quand, Comment, Pourquoi, 2009 [On-line]. Disponible sur : http://qualite-enrecherche.cnrs.fr/spip.php?article6

8. Chartier S, Ferrat E, Djassibel M, Bercier S, Compagnon L, Renard V et al. Mise en ouvre d'un programme d'apprentissage dans une logique de compétence : difficultés et propositions. Exercer 2012;103:169-74.
9. Rodger S, Hoffman S. On behalf of the World health organisation study on interprofessionnal education and collaborative pratice. Where in the world is interprofessional education ? A global environmental scan. J Interprof Care 2010;24:479-91.

10. Fiquet L, Huge S, Annezo F, Chapron A, Allory E, Renaut P. Une formation inter professionnelle pour apprendre à travailler ensemble. La perception des étudiants en santé. PédagogieMédicale 2015 http:// dx.doi.org/10.1051/pmed/2015018

11. Jouquan J, Bail P. A quoi s'engage-t-on en basculant du paradigme d'enseignement vers le paradigme d'apprentissage ? Pédagogie Médicale 2003;4:163-75.

12. Attali C, Huez J, Valette T. Les grandes familles de situations cliniques. Exercer 2013;24:165-69.

13. Association nationale des étudiants en médecine de France (ANEMF) \& Intersyndicale nationale autonome représentative des internes de médecine générale (ISNAR-IMG). Démographie Médicale Contribution commune. 2011 [On-line]. Disponible sur : http://static.hospimedia.fr/documents/00000/ 110620ISNARIMGANEMF.pdf

14. Conseil National de l'ordre des médecin, Legmann M, Ramestaing P, Le Breton-Lerouvillois G. Atlas de la démographie médicale en France - Situation au 1er janvier 2013. Paris : Ordre National des Médecins. 2013 [On-line]. Disponible sur : https:// www.conseil-national.medecin.fr/sites/default/files/ atlas_national_2013.pdf

15. Lévy D. Le métier de médecin aujourd'hui. Revue Française des Affaires Sociales 2011;2:297-309.

Charline Boissy, Département de médecine générale, Faculté de Médecine Lyon Est, 8 avenue Rockefeller, 69373 Lyon Cedex, France.

Mailto : Charline.boissy@gmail.com 